\title{
The CMS Event-Builder System for LHC Run 3 (2021-23)
}

Jean-Marc André ${ }^{5}$, Ulf Behrens ${ }^{1}$, James Branson ${ }^{4}$, Philipp Brummer ${ }^{2,11}$, Sergio Cittolin ${ }^{4}$, Diego Da Silva Gomes ${ }^{2}$, Georgiana-Lavinia Darlea ${ }^{6}$, Christian Deldicque ${ }^{2}$, Zeynep Demiragli $^{6}$, Marc Dobson ${ }^{2}$, Nicolas Doualot ${ }^{5}$, Samim Erhan $^{3}$, Jonathan Fulcher ${ }^{2}$, Dominique Gigi $^{2}$, Maciej Gładki ${ }^{2}$, Frank Glege ${ }^{2}$, Guillelmo Gomez-Ceballos ${ }^{6}$, Jeroen Hegeman ${ }^{2}$, André Holzner ${ }^{4}$, Michael Lettrich ${ }^{2}$, Audrius Mečionis ${ }^{5,9}$, Frans Meijers ${ }^{2}$, Emilio Meschi ${ }^{2}$, Remigius K Mommsen ${ }^{5, *}$, Srećko Morović5 ${ }^{\text {, Vivian O’Dell }}{ }^{5}$, Luciano Orsini ${ }^{2}$, Ioannis Papakrivopoulos ${ }^{7}$, Christoph Paus $^{6}$, Andrea Petrucci ${ }^{8}$, Marco Pieri ${ }^{4}$, Dinyar Rabady ${ }^{2}$, Attila Rácz $^{2}$, Valdas Rapševičius ${ }^{5,9}$, Thomas Reis ${ }^{2}$, Hannes Sakulin ${ }^{2}$, Christoph Schwick ${ }^{2}$, Dainius Šimelevičius ${ }^{2,9}$, Mantas Stankevičius ${ }^{5,9}$, Cristina Vazquez Velez $^{2}$, Christian Wernet $^{2}$, and Petr Zejdl ${ }^{5,10}$

${ }^{1}$ DESY, Hamburg, Germany

${ }^{2}$ CERN, Geneva, Switzerland

${ }^{3}$ University of California, Los Angeles, Los Angeles, California, USA

${ }^{4}$ University of California, San Diego, San Diego, California, USA

${ }^{5}$ FNAL, Batavia, Illinois, USA

${ }^{6}$ Massachusetts Institute of Technology, Cambridge, Massachusetts, USA

${ }^{7}$ Technical University of Athens, Athens, Greece

${ }^{8}$ Rice University, Houston, Texas, USA

${ }^{9}$ Also at Vilnius University, Vilnius, Lithuania

${ }^{10}$ Also at CERN, Geneva, Switzerland

${ }^{11}$ Also at Karlsruhe Institute of Technology, Karlsruhe, Germany

Abstract. The data acquisition system (DAQ) of the CMS experiment at the CERN Large Hadron Collider (LHC) assembles events of $2 \mathrm{MB}$ at a rate of $100 \mathrm{kHz}$. The event builder collects event fragments from about 750 sources and assembles them into complete events which are then handed to the High-Level Trigger (HLT) processes running on $O(1000)$ computers. The aging eventbuilding hardware will be replaced during the long shutdown 2 of the LHC taking place in 2019/20. The future data networks will be based on $100 \mathrm{~Gb} / \mathrm{s}$ interconnects using Ethernet and Infiniband technologies. More powerful computers may allow to combine the currently separate functionality of the readout and builder units into a single $\mathrm{I} / \mathrm{O}$ processor handling simultaneously $100 \mathrm{~Gb} / \mathrm{s}$ of input and output traffic. It might be beneficial to preprocess data originating from specific detector parts or regions before handling it to generic HLT processors. Therefore, we will investigate how specialized coprocessors, e.g. GPUs, could be integrated into the event builder. We will present the envisioned changes to the event-builder compared to today's system. Initial measurements of the performance of the data networks under the event-building traffic pattern will be shown. Implications of a folded network architecture for the event building and corresponding changes to the software implementation will be discussed.

\footnotetext{
*Corresponding author: remigius.mommsen@cern.ch
} 


\section{Introduction}

The Compact Muon Solenoid (CMS) experiment at CERN is one of the two general purpose experiments located at the LHC. CMS is designed to study both proton-proton and heavy ion collisions at the $\mathrm{TeV}$ scale [1]. The detector comprises about 55 million readout channels. The online event-selection is performed using two trigger levels: a hardware-based first-level (L1) trigger accepting up to $100 \mathrm{kHz}$ of events and a software-based High-Level Trigger (HLT) selecting $O(1 \%)$ of these events.

The Run-2 event-builder system [2] collects event fragments from about 750 detector backend boards (FEDs) at the L1 trigger rate. It transports the fragments over about $200 \mathrm{~m}$ to the surface using a simplified TCP/IP protocol [3] over 10 and $40 \mathrm{~Gb} / \mathrm{s}$ Ethernet to a predefined Readout Unit (RU) computer. The RU splits the streams into event fragments, checks the consistency and buffers them until it receives the assignment message from the event manager (EVM). Once the RU knows which Builder Unit (BU) machine has been assigned to handle the event, the RU combines all fragments belonging to the same event into a superfragment. Each RU waits until it has received the fragments from all FEDs allocated to it before creating the super-fragment. The super-fragment is sent over the event-builder switch to the BUs. The event-builder switch uses Infiniband [4] FDR at $56 \mathrm{Gbit} / \mathrm{s}$. The BU assembles the super-fragments into complete events and writes them to a local RAMdisk from where they are picked up by the file-base filter farm [5].

\section{The DAQ System for LHC Run 3}

The LHC undergoes a 2-year maintenance period during 2019/20, followed by another 3 years of running. The conditions for the next running period (Run 3) will be similar to Run 2 . There will be a few modifications to the CMS detector which results an a slight increase in the number of readout channels and event size. However, the commercial equipment of the current DAQ system reaches end-of-life after 5 years and needs to be replaced. We will use this opportunity to keep abreast with technological evolution, and explore solutions for the next generation DAQ system [6, 7] needed for the high-luminosity LHC [8] planned for 2025. Novel solutions to be looked at are the combination of the currently separate readout and builder units into a single I/O processor, an architecture known as 'folded.' The integration of co-processors (GPUs and/or FPGAs) into the event building to pre-process events before handing them to the HLT and partial event acquisition at the bunch-crossing rate of $40 \mathrm{MHz}$ are further topics which will be looked into.

The main enabler for smaller and more efficient DAQ systems are network interconnects. Figure 1 shows the evolution of the market share of network interconnects used in the 500 most performing super-computers. The DAQ system for Run $1[9,10]$ conceived in 2002 used two $2 \mathrm{~Gb} / \mathrm{s}$ Myrinet links between the backend electronics and the RU, and several $1 \mathrm{~Gb} / \mathrm{s}$ Ethernet connections between the RU and BU. It required $640 \mathrm{RUs}$ to handle $1 \mathrm{MB}$ events at $100 \mathrm{kHz}$ trigger rate. The event building was organized into 8 separate readout slices each providing a throughput of about $12.5 \mathrm{~GB} / \mathrm{s}$. The events were assigned to the slices in a roundrobin scheme. The Run-2 DAQ system built in 2014 replaced Myrinet with 10 and $40 \mathrm{~Gb} / \mathrm{s}$ Ethernet and uses Infiniband FDR $(56 \mathrm{~Gb} / \mathrm{s})$ as the event-builder network. This allowed to shrink the size of the system by an order of magnitude, while providing twice the throughput required to handle event sizes of up to $2 \mathrm{MB}$ at $100 \mathrm{kHz}$ trigger rate. The DAQ system for Run 3 continues to use Ethernet and Infiniband, but exploits the faster Ethernet and Infiniband link speeds of $100 \mathrm{~Gb} / \mathrm{s}$ easily available today. The main change will be the combination of the functionality of the readout and builder units into a single machine as described in the next section. The DAQ system for Run 3 will be used to gain experience with this architecture 


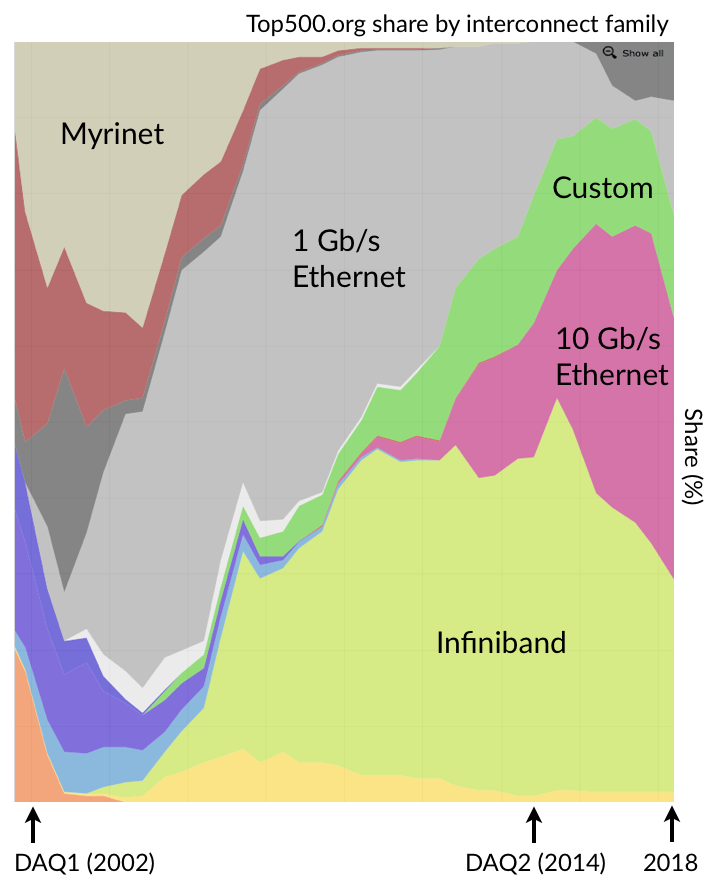

Figure 1. Evolution of market share of network interconnects used in the 500 most performing super-computers from 2000 to 2018.

which is needed for the high-luminosity LHC, where the DAQ system will need to handle $5.5 \mathrm{~TB} / \mathrm{s}$ using about $500 \mathrm{I} / \mathrm{O}$ nodes. The event size at high-luminosity LHC will increase to about $7.4 \mathrm{MB}$ and the trigger rate goes up to $750 \mathrm{kHz}$.

\section{Folded Event-Builder Architecture}

Figure 2 shows a simplified schematic of the current DAQ system and of a possible folded configuration. The readout from the detector backends remains unchanged. It will still be based on the same custom electronics boards sending the data over $10 \mathrm{~Gb} / \mathrm{s} \mathrm{TCP} / \mathrm{IP}$ streams. These streams will be concentrated into $100 \mathrm{~Gb} / \mathrm{s}$ instead of $40 \mathrm{~Gb} / \mathrm{s}$ Ethernet links to the Readout Units (RUs). The functionality of the fragment buffering on the RUs and the full event building in the Builder Units (BUs) will be collapsed into I/O nodes. These nodes are interconnected by EDR Infiniband at $100 \mathrm{~Gb} / \mathrm{s}$. The HLT nodes will share the $100 \mathrm{~Gb} / \mathrm{s}$ Ethernet network interface cards to access the fully built events. The folded architecture allows to exploit the bi-directional bandwidth of the network links. It requires about half the number of machines and switch ports, which reduces the cost of the system. This is especially important for the DAQ system to be built for the high-luminosity LHC, which will need to handle a 30 times higher throughput. The main challenge of a folded architecture is the demanding $\mathrm{I} / \mathrm{O}$ and memory performance of the $\mathrm{I} / \mathrm{O}$ node. It has to process TCP/IP streams at $100 \mathrm{~Gb} / \mathrm{s}$, distribute the event fragments to other builder units at $100 \mathrm{kHz}$, build complete events at $1-2 \mathrm{kHz}$ using fragments received from all other nodes, and make the complete events available to the HLT processors. In addition, it may also need to hand events to co-processors and receive the results of the calculations.

In order to assess the feasibility of a folded event-builder architecture, we use the current DAQ system with the full event-builder software. The event-building applications are built upon the XDAQ framework [11]. XDAQ is a middleware that eases the development of distributed data acquisition systems. The framework has been developed at CERN and builds 


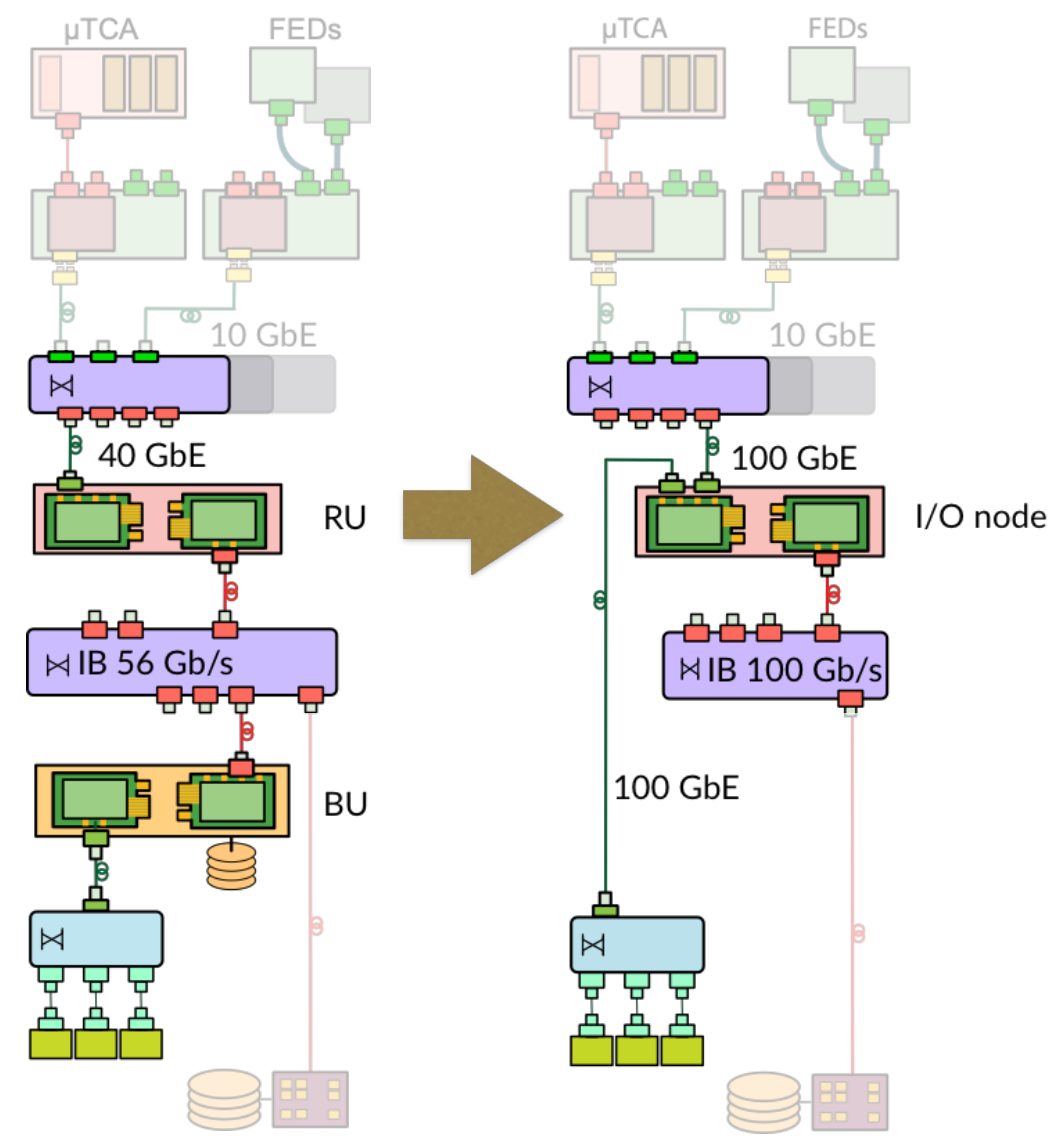

Figure 2. A simplified schematic of the current DAQ system (left) and a possible folded scheme for the DAQ system for Run 3 (right). The main change will be that the Readout Unit (RU) and the Builder Unit (BU) executables will no longer be deployed on separate machines but on a common I/O node.

upon industrial standards, open protocols and libraries. It provides services for data transport, configuration, monitoring, and error reporting. In particular, it also provides a zero-copy architecture for Infiniband [12]. The flexible configuration scheme provided by the XDAQ framework allows to combine the RU and BU functionality without any software change. Only the NUMA settings were re-tuned for the folded configuration to optimize the pinning to CPU cores and memory allocation.

The RU and BU nodes of the Run-2 production system are Dell R620 and R720 dual 8-core sandy bridge @ $2.6 \mathrm{GHz}$, respectively. They are interconnected with a Clos-network structure with 12 leaf and 6 spine switches (Mellanox SX6036), providing 216 external ports and $12 \mathrm{Tbit} / \mathrm{s}$ bi-sectional bandwidth. For this test, event fragments are generated on the readout unit, i.e. they are not received from TCP/IP streams. Fully built events are discarded on the builder unit instead of being written out. We measured the throughput using a productionlike setup where 73 RUs send the data to 73 BUs, and the folded architecture were the BU is running on the same node as the RU. Figure 3 shows that the plateau throughput of the folded architecture is reduced by $\sim 15 \%$ compared to the standard unidirectional configuration. The 


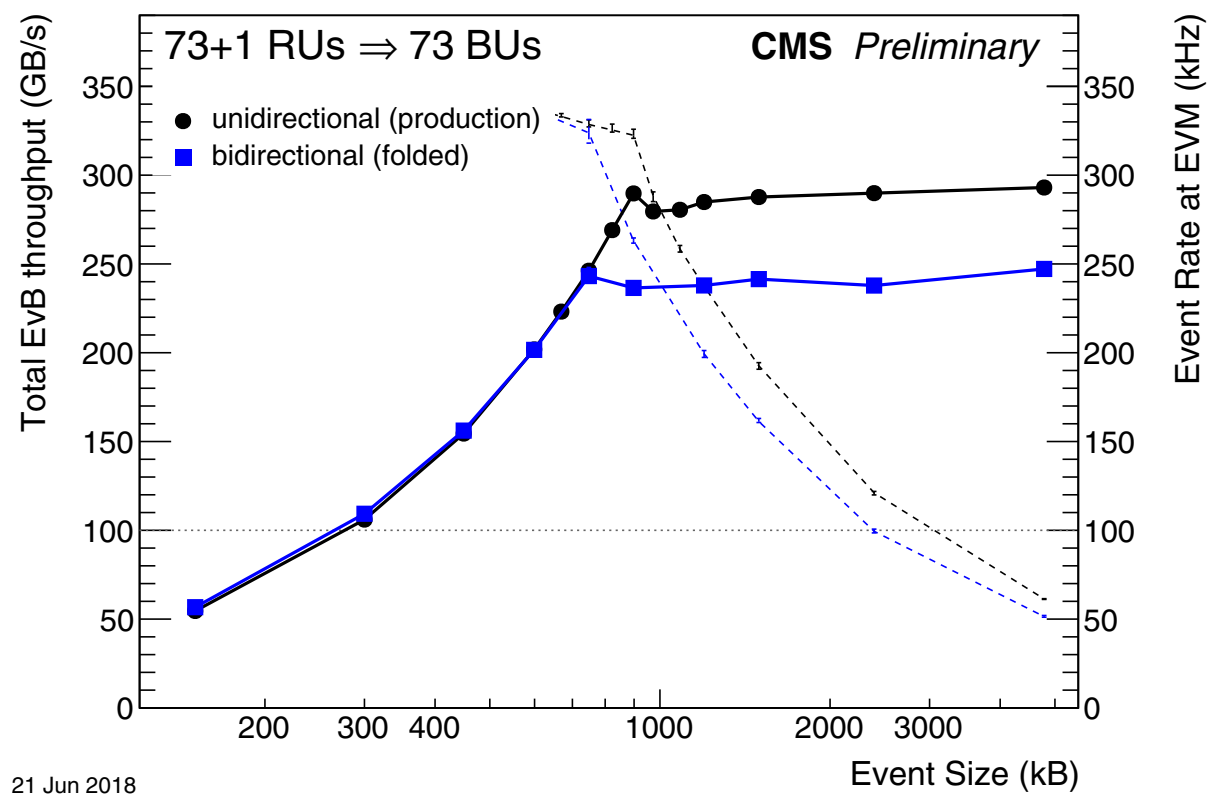

Figure 3. Performance measurement of the event-builder system using the current production system for the standard unidirectional traffic, and for a folded (bidirectional) configuration. The thick lines show the total event-building throughput as function of the event size (left axis). The dashed lines show the equivalent trigger rate in $\mathrm{kHz}$ (right axis). The point where they go below the requirement of $100 \mathrm{kHz}$ (dotted line) indicates the maximum event size which can be handled.

folded configuration suffers from the additional $\mathrm{I} / \mathrm{O}$ requirements and from the large number of threads, which exceed the CPU core count of the legacy hardware. Nevertheless, $2 \mathrm{MB}$ events could still be built at $100 \mathrm{kHz} \mathrm{L1}$ trigger rate.

The folded architecture was also tested on a small-scale test system which is based on state-of-the-art hardware with 16 Dell R740 dual 16-cores skylake @ 2.1/2.6 GHz. They are interconnected with a single Mellanox MSB7800 EDR switch (100 Gb/s). Figure 4 shows that an up to 3 times higher throughput is achievable compared to current production hardware using a single leaf switch of the Clos network. Preliminary measurements where the BU writes the data to a RAM-disk show that the disk-writing limits the throughput per BU to $\lesssim 5.5 \mathrm{~GB} / \mathrm{s}$. Therefore, the distributions of events to the HLT needs to be improved in order to make use of the higher throughput achievable when building events only.

\section{Event Building with MPI}

The successful use of the same network equipment in the CMS event builder that is found in a majority of today's supercomputers raises the question, if synergies between HPC software APIs and event building exist and how they could be exploited. A feasibility study [13] reviewed the Messages Passing Interface (MPI) [14], a framework used in HPC for developing multi-process applications that operate asynchronously on different sets of data. The high level, hardware agnostic API specification is implemented and tuned by vendors to support support various configurations from same machine shared memory to high-performance interconnects such as Infiniband or Omnipath, promising highly portable application code. 


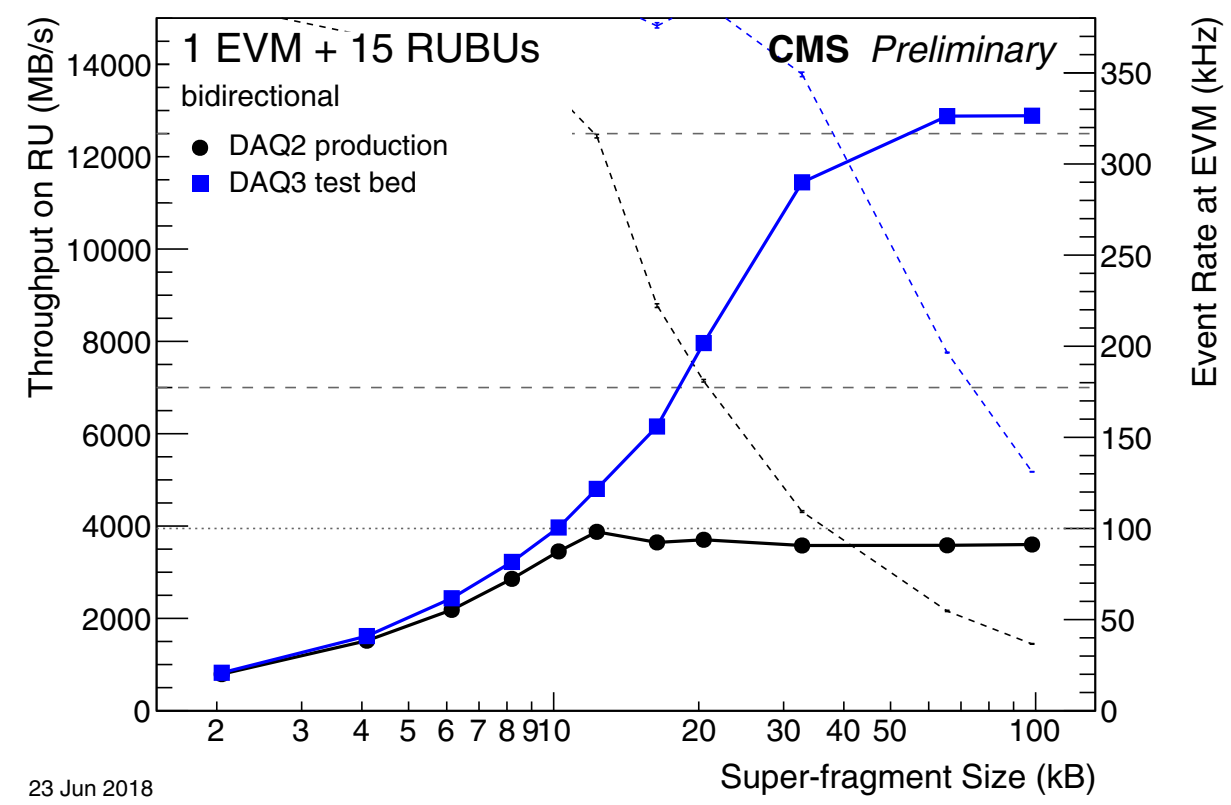

Figure 4. Measurement of a folded event-builder architecture with 15 nodes using the current production hardware (Run 2), and state-of-the-art hardware installed in a test bed for the next generation DAQ system for Run 3. The thick lines show the throughput on the Readout Unit (RU) as function of the super-fragment size generated on the RU. The dashed lines show the equivalent trigger rate in $\mathrm{kHz}$ (right axis).

The API defines communication operations suitable for a large variety of use cases, that can be separated into three groups. Point-to-point communication allows to exchange data between two processes using explicit send and receive operations. Collective communication procedures implement data exchange patterns along a group of processes. One-sided communication also referred to as Remote Memory Access (RMA) allows direct access of memory previously exposed by another process.

In the end only MPI point-to-point communication procedures provided the necessary flexibility and fault tolerance. The collective procedures proved to be inflexible when dealing with non-uniform fragment sizes and error handling. One-sided procedures, which decouple the exchange of data from synchronization, could not be mapped to the problem of event building efficiently.

The performance of MPI and Infiniband Verbs used by XDAQ has been evaluated using a benchmark, that simulates two stage event building traffic without building the actual events. Highest throughput was reached using a zero-copy queuing algorithm based on MPI pointto-point communication. MPI Runtime parameters enforced data locality between communication processes and Infiniband hardware and network transport was delegated by the MPI API to the vendor tuned UCX [15] framework. Despite our efforts, only a slightly higher performance than with Infiniband Verbs was achieved (see Figure 5). The marginal performance gain, combined with limited fault tolerance and large tuning efforts for MPI, leads to the conclusion that as of now, the MPI API does not map onto the task of event building at the CMS experiment. 


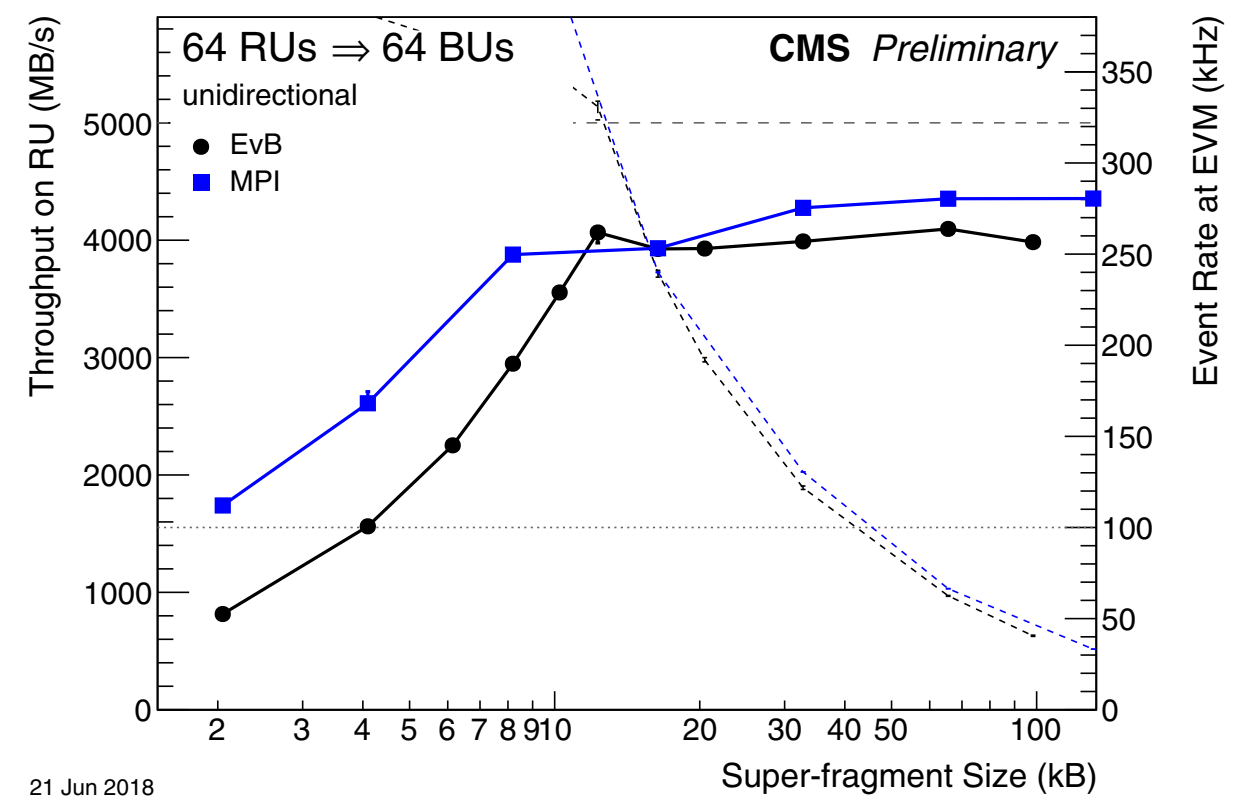

Figure 5. Comparison of the throughput of the full event-buidler software (EvB) with a simple eventbuilder benchmark based on MPI point-to-point communication. The MPI benchmark shows a better performance for small super-fragment sizes, but does not achieve a significant improvement for larger sizes.

\section{Integration of Co-Processors}

The integration of general-purpose GPUs and/or FPGAs into the reconstruction of events might be a cost-effective solution to reduce the required CPU power on the High-Level Trigger (HLT). This will become a real issue on the time scale of the high-luminosity LHC. Equipping each HLT node with a GPU could not be cost effective. The GPU cannot be fully loaded unless the majority of the event reconstruction and selection can be done on the GPU. In addition, the life cycle of the GPU and host computer might be different, which complicates any hardware upgrades.

A solution would be to offload specific parts of the event reconstruction to a farm of GPU-equipped nodes during the HLT processing. However, this requires a mechanism and network to transfer data to and from a GPU farm. This adds latency for data transport and careful tuning will be needed to avoid that the HLT CPUs stall on network transfers.

Another option would be to pre-process all events at the event-builder level before handing them to the HLT farm. This avoids any latency during the HLT processing and is technically easier to solve. It could be an effective solution for specific tasks on a subset of the event data, e.g. to reconstruct tracks in the pixel detector. However, such an approach would waste many GPU cycles if the result from the calculation is only used for a small number of events.

In any case, the Run-3 DAQ system could be a good test bed to investigate technical solutions, and to learn about issues which need to be taken into account for the DAQ system to be designed for the High-Luminosity LHC. 


\section{Summary}

The requirements of the DAQ system for the LHC Run 3 in 2021-23 do not change significantly. However, the commercial components of the current DAQ system reach their end of life after more than 5 years. Therefore, a new DAQ system needs to be built in 2020. It will make use of $100 \mathrm{~Gb} / \mathrm{s}$ Ethernet and Infiniband networks, as well as more powerful PCs.

The DAQ system for Run 3 will be used to gain experience with techniques needed for the much more performant DAQ system needed for high-luminosity LHC. An interesting option is the use of a folded architecture, which would better exploit the hardware capabilities. Results from initial measurements using the Run-2 production system and a testbed featuring state-of-the-art hardware look promising. However, handling a $100 \mathrm{~Gb} / \mathrm{s} \mathrm{TCP} / \mathrm{IP}$ stream on the PC in addition to the full event-building traffic will be challenging. Special attention is needed to find an efficient working point in terms of fragment sizes. In addition, the data distribution to the HLT farm needs to be improved in order to be able to shrink the size of the DAQ system.

We plan to investigate how GPU and/or FPGAs could be integrated into the online event reconstruction either at the event-builder level or during the HLT processing. Investigations into using MPI for the event building did not show any clear benefit over the current verbsbased implementation. However, MPI could be an option to ease the integration of GPUs into the event building.

\section{Acknowledgments}

This work was supported in part by the DOE and NSF (USA).

\section{References}

[1] CMS Collaboration, JINST 3, S08004 (2008)

[2] R.K. Mommsen et al., J. Phys. Conf. Ser. 898, 032020 (2017)

[3] P. Zejdl et al., J. Phys. Conf. Ser. 513, 012042 (2014)

[4] InfiniBand Trade Association, see http://www.infinibandta.com

[5] E. Meschi et al., J. Phys. Conf. Ser. 664, 082033 (2015)

[6] E. Meschi et al., J. Phys. Conf. Ser. 898, 032019 (2017)

[7] J. Hegeman et al. (2018), arXiv 1806.08975

[8] G. Apollinari, I. Béjar Alonso, O. Brüning, P. Fessia, M. Lamont, L. Rossi, L. Tavian, High-Luminosity Large Hadron Collider (HL-LHC): Technical Design Report V. 0.1, CERN Yellow Reports: Monographs (CERN, Geneva, 2017), http: //cds.cern.ch/ record/2284929

[9] G. Bauer et al., IEEE Trans. Nucl. Sci. 55, 198 (2008)

[10] R.K. Mommsen et al., J. Phys. Conf. Ser. 219, 022038 (2010)

[11] J. Gutleber et al., J. Phys. Conf. Ser. 219, 022011 (2010)

[12] A. Petrucci et al., IEEE Trans. Nucl. Sci. 60, 4595 (2013)

[13] M. Lettrich, Master's thesis, CERN and Technical University of Munich (2018), https://mediatum.ub.tum.de/1482023

[14] Message Passing Interface Forum, MPI: A Message-Passing Interface Standard, Version 3.1 (High Performance Computing Center Stuttgart (HLRS), 2015)

[15] P. Shamis et al., UCX: An Open Source Framework for HPC Network APIs and Beyond, in 2015 IEEE 23rd Annual Symposium on High-Performance Interconnects (2015), pp. 40-43, ISSN 1550-4794 